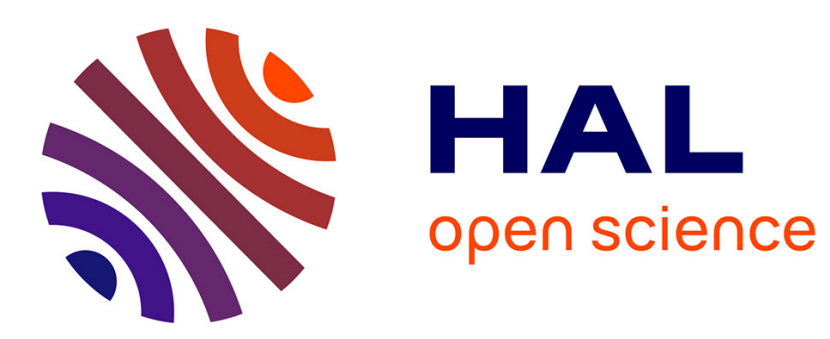

\title{
Calculs des niveaux d'énergie liés et quasi-liés des ions moléculaires muoniques $\mathrm{He} \mu+$ et $\mathrm{Ne} \mu+$ \\ P.G. Fournier, B. Lassier-Govers
}

\section{To cite this version:}

P.G. Fournier, B. Lassier-Govers. Calculs des niveaux d'énergie liés et quasi-liés des ions moléculaires muoniques $\mathrm{He} \mu+$ et $\mathrm{Ne} \mu+$. Journal de Physique Lettres, 1982, 43 (13), pp.483-491. 10.1051/jphyslet:019820043013048300 . jpa-00232080

\section{HAL Id: jpa-00232080 https://hal.science/jpa-00232080}

Submitted on 1 Jan 1982

HAL is a multi-disciplinary open access archive for the deposit and dissemination of scientific research documents, whether they are published or not. The documents may come from teaching and research institutions in France or abroad, or from public or private research centers.
L'archive ouverte pluridisciplinaire HAL, est destinée au dépôt et à la diffusion de documents scientifiques de niveau recherche, publiés ou non, émanant des établissements d'enseignement et de recherche français ou étrangers, des laboratoires publics ou privés. 
Classification

Physics Abstracts

$33.10-36.10$

\title{
Calculs des niveaux d'énergie liés et quasi-liés des ions moléculaires muoniques $\mathrm{He}^{+}$et $\mathrm{Ne} \mu^{+}$
}

\author{
P. G. Fournier et B. Lassier-Govers \\ L.C.A.M. (*), Groupe de Spectroscopie de Translation, \\ Bâtiment 470, Université de Paris-Sud, 91405 Orsay, France
}

(Reçu le 26 janvier 1982, révisé le 29 mars, accepté le 10 mai 1982)

\begin{abstract}
Résumé. - A partir des courbes de potentiel des systèmes $\mathrm{HeH}^{+}$et $\mathrm{NeH}^{+}$, on a calculé les niveaux d'énergie rovibrationnels des ions moléculaires mésiques correspondants $\left(\mathrm{He} \mu^{+}\right.$et $\left.\mathrm{Ne} \mu^{+}\right)$. On a aussi déterminé les énergies des états quasi-liés, la hauteur des barrières de potentiel correspondantes ainsi que les durées de vie de ces états vis-à-vis de la prédissociation. Les contributions des corrections diabatiques et adiabatiques à ces résultats sont discutées.
\end{abstract}

\begin{abstract}
Bound and quasi-bound states of mesic molecular ions have been calculated in the framework of the Born-Oppenheimer approximation. The energy levels, potential humps, and lifetimes with respect to predissociation of $\mathrm{He} \mu^{+}$and $\mathrm{Ne} \mu^{+}$quasi-bound states are determined. Diabatic and non adiabatic corrections are estimated and discussed.
\end{abstract}

La physique atomique et moléculaire des muons traite essentiellement les interactions des $\mu^{-}$ avec la matière; les forces et structures nucléaires et atomiques sont profondément modifiées quand un atome ou une molécule muonique est formé lors de la substitution d'un électron par un muon négatif [1]. Cependant dès 1957 [2], l'existence d'un autre type de molécules transitoires formées à partir d'un muon positif avait été envisagée. En effet le muon $\mu^{+}$de spin $\frac{1}{2}$, de masse 0,1132920 u.m.a., peut être considéré comme un isotope instable du proton ayant une durée de vie $2,2 \mu \mathrm{s}$.

Dans ce type de molécule, le muon positif ou le muonium remplace un proton ou un atome d'hydrogène de la même façon que le muon négatif se substituait à un électron [3]. Bien que le muonium $\left(\mu^{+} \mathrm{e}^{-}\right)$ait été mis en évidence directement dès 1960 [4], il n'existe aucune preuve expérimentale directe, obtenue par spectrométrie optique ou de masse, de l'existence de telles molécules « exotiques ». Cependant toute une chimie de ces molécules muoniques se développe actuellement près des grands accélérateurs [5]. L'analyse des réactions muoniques se fait essentiellement à l'aide de deux techniques basées sur la propriété caractéristique des faisceaux de muons d'être polarisés (à près de $90 \%$ ) longitudinalement lors de leur production. Dans la première approche dite $\mu$.S.R. (Muon. Spin. Resonance) le faisceau de muons est thermalisé par collision à l'intérieur de la cible diamagnétique placée dans un champ magnétique perpendiculaire

(*) Laboratoire associé au C.N.R.S. (LA 0281). 
à la direction de polarisation initiale du spin du muon. Le spin des muons précesse alors à la fréquence de Larmor du muon libre : $\omega(\mu)=0,0136 \mathrm{MHz} / \mathrm{G}$. La deuxième technique dite M.S.R. (Muonium. Spin. Resonance) utilise la précession de l'atome muonique $\left(\mu^{+} \mathrm{e}^{-}=\mathrm{Mu}\right)$. Le muonium est formé dans des états singulet et triplet. Son moment magnétique est sensiblement le même que celui de l'électron. La précession du muonium dans l'état triplet sera donc 103 fois plus rapide que celle du muon libre : $\omega(\mathrm{Mu})=1,29 \mathrm{MHz} / \mathrm{G}$. Dans les deux cas, l'histogramme de la précession du muon est obtenu en détectant le positron de désactivation qui est émis préférentiellement dans la direction du spin du muon. Toute dépolarisation du signal est généralement interprétée comme la conséquence d'un couplage avec la cible.

Le problème posé est de savoir si le $\mu^{+}$se stabilise dans le milieu cible en tant que muon libre, muonium, molécule ou ion moléculaire. La compréhension des mécanismes de relaxation de l'énergie des muons épithermiques, et l'observation éventuelle par spectroscopie optique [6] de molécules muoniques présupposent la détermination des caractéristiques des états rovibrationnels.

Suite à nos travaux sur $\mathrm{HeH}^{+}$[7] et aux travaux de Fleming [8], nous nous sommes intéressés à la formation d'ions moléculaires muoniques de gaz rares. T. W. Crane et al. [9] avaient déterminé expérimentalement les précessions du muon, du muonium et la dépolarisation de spin lors de l'interaction avec une cible d'hélium liquide. Les résultats de leurs travaux n'excluaient pas la formation de molécules $\mathrm{He} \mu^{+}$ou d'agrégats d'atomes d'hélium englobant le muon. Dans le cas de l'hélium, ainsi que l'avaient montré Stambough et al. [10], la formation de l'ion muonique crée une très faible dépolarisation de l'ordre de 1 p.p.m. Plus récemment Mikula et al. [11] ont repris systématiquement ces expériences en phase gazeuse à basse pression (1 bar). Ces études ont été effectuées pour tous les gaz rares ainsi que pour des mélanges dans lesquels une faible concentration de néon, de krypton et de xénon était introduite à titre d'impuretés. Les divers spectres de précession, remarquablement résolus, obtenus à diverses températures, montrent d'incontestables effets de dépolarisation en particulier pour le néon. Ces dépolarisations suggèrent la formation d'ions moléculaires. L'interprétation de ces travaux implique la compréhension des mécanismes de formation des ions moléculaires ainsi que la détermination des niveaux d'énergie rovibroniques. Différents auteurs ont alors calculé les niveaux d'énergie liés de la molécule $\mathrm{HMu}$ [6], des ions $\mathrm{He} \mu^{+}$et $\mathrm{Ne} \mu^{+}[11 a$ ] en se plaçant dans l'approximation de l'oscillateur harmonique, les énergies de vibration des molécules isotopiques étant inversement proportionnelles aux masses réduites. Nous avons déterminé les niveaux d'énergies des états liés ainsi que les énergies et les durées de vie des états quasi-liés piégés dans la barrière centrifuge en résolvant la partie radiale de l'équation de Schrödinger.

L'importance considérable, pour la chimie et la physique des interactions moléculaires, de la connaissance des propriétés des états quasi-liés a été soulignée dans un article de revue présenté par Child [12]. Nos calculs ont été effectués dans le cadre de l'approximation de Born-Oppenheimer qui découple les mouvements de vibration et de rotation des noyaux de celui des électrons. Les calculs ab initio de courbes de potentiel de Kotos et Peek [13] pour $\mathrm{HeH}^{+}$et Rosmus [14] pour $\mathrm{NeH}^{+}$ne font pas intervenir de forces dépendant des masses, le proton est considéré comme une charge ponctuelle fixe. On peut donc, malgré le déplacement du centre de masse, utiliser ces mêmes courbes pour $\mathrm{He}^{+}$et $\mathrm{Ne} \mu^{+}$. Les potentiels effectifs pour chaque nombre quantique $J$ sont obtenus en ajoutant l'énergie de rotation $J(J+1) / 2 m R^{2}$. La masse réduite $m$ de ces molécules muoniques étant faible, les énergies centrifuges auront une contribution plus importante que dans le cas des molécules protoniques. Ayant admis que le muon se comporte essentiellement comme un isotope léger du proton on peut utiliser les mêmes programmes de calculs que pour les molécules protonées. Les niveaux d'énergie liés sont déterminés numériquement grâce à un algorithme de type Numerov. La difficulté dans la détermination des états quasi-liés est due au couplage d'un état discret avec un continuum et à la détermination du comportement asymptotique de la fonction d'onde. Les diverses méthodes de calculs, longues et d'applications délicates, des résonances situées 
dans la barrière de potentiel sont décrites en utilisant les diverses propriétés des fonctions d'ondes. Dans le cas des potentiels sphériques R. J. Le Roy et W. K. Liu [15] ont comparé les différentes méthodes de calculs et ont montré que les désaccords sur la détermination des positions énergétiques des états rovibrationnels étaient négligeables et qu'ils n'étaient sensibles que sur les largeurs de ces états $\left(\simeq 20 \%\right.$ ). Dans le cas de $\mathrm{HeH}^{+}$nous avions déterminé les énergies des états quasi-liés et leurs largeurs en étudiant le déphasage de la fonction d'onde : chaque résonance en énergie est déterminée lorsque la phase change de $\pi$, la largeur de l'état correspond à la variation d'énergie associée à un changement de $\pi / 2$ de la phase. Les résultats diffèrent de moins de cinq pour dix mille

Tableau I. - Energies $W$ des niveaux rovibrationnels des ions moléculaires $\mathrm{He} \mu^{+}$et $\mathrm{Ne} \mu^{+}$. Les énergies $(e n \mathrm{eV})$ sont repérées par rapport aux limites de dissociation $: \mu^{+}+\mathrm{He}, \mu^{+}+\mathrm{Ne}$.

[Energies $W$ of the rovibrational levels of the molecular ions $\mathrm{He} \mu^{+}$and $\mathrm{Ne} \mu^{+}$. They are quoted (in $\mathrm{eV}$ ) relative to the dissociation limits $\left(\mu^{+}+\mathrm{He}\right)$ and $\left(\mu^{+}+\mathrm{Ne}\right)$, respectively.]

\begin{tabular}{|c|c|c|c|c|c|c|c|c|c|c|}
\hline \multicolumn{6}{|c|}{$\underline{\mathrm{He}}^{+}$} & \multicolumn{5}{|c|}{$\underline{\mathrm{Ne}}^{+}$} \\
\hline & 0 & 1 & 2 & 3 & & 0 & 1 & 2 & 3 & 4 \\
\hline $\begin{array}{l}0 \\
1 \\
2 \\
3 \\
4 \\
5 \\
6 \\
7 \\
8 \\
9\end{array}$ & $\begin{array}{r}-1,53251 \\
-1,47647 \\
-1,36687 \\
-1,20848 \\
-1,00804 \\
-0,77401 \\
-0,51622 \\
-0,24606 \\
0,02235 \\
0,26414\end{array}$ & $\begin{array}{r}-0,73430 \\
-0,69189 \\
-0,60954 \\
-0,49210 \\
-0,34677 \\
-0,18330 \\
-0,01565 \\
0,13014\end{array}$ & $\begin{array}{l}-0,22408 \\
-0,19716 \\
-0,14632 \\
-0,7795 \\
-0,00384\end{array}$ & $\begin{array}{r}-0,01753 \\
-0,00928 \\
0,00192\end{array}$ & $\begin{array}{r}0 \\
1 \\
2 \\
3 \\
4 \\
5 \\
6 \\
7 \\
8 \\
9 \\
10 \\
11 \\
12\end{array}$ & $\begin{array}{r}-1,78632 \\
-1,75235 \\
-1,68497 \\
-1,58529 \\
-1,45499 \\
-1,29626 \\
-1,11183 \\
-0,90496 \\
-0,67946 \\
-0,43978 \\
-0,19121 \\
0,05951 \\
0,30134\end{array}$ & $\begin{array}{r}-0,97497 \\
-0,94760 \\
-0,89344 \\
-0,81365 \\
-0,71001 \\
-0,58490 \\
-0,44142 \\
-0,28352 \\
-0,11640 \\
0,05228 \\
0,20707\end{array}$ & $\begin{array}{r}-0,40255 \\
-0,38239 \\
-0,34279 \\
-0,28518 \\
-0,21185 \\
-0,12621 \\
-0,03338 \\
0,05609\end{array}$ & $\begin{array}{r}-0,08362 \\
-0,07229 \\
-0,05079 \\
-0,02188 \\
0,00798\end{array}$ & $\begin{array}{l}-0,00229 \\
-0,00030\end{array}$ \\
\hline
\end{tabular}

Tableau II. - Valeurs des paramètres caractéristiques des niveaux rovibrationnels quasi-liés des ions moléculaires $\mathrm{He}^{+}$et $\mathrm{Ne} \mu^{+}: v, J$ : nombres quantiques de vibration et rotation; $W$ : énergie du niveau par rapport à la limite de dissociation; $\tau$ : durée de vie calculée; $h:$ hauteur de la barrière de potentiel.

[Characteristic parameters for the quasi-bound rovibrational levels of the molecular ions $\mathrm{He} \mu^{+}$ and $\mathrm{Ne} \mu^{+}: v$ and $J$ are the vibrational and rotational quantum numbers; $W$ is the energy above the dissociation limit; $\tau$ is the calculated lifetime, and $h$ the height of the effective-potential barrier.]

$$
\mathrm{He} \mu^{+}
$$

$\mathrm{Ne} \mu^{+}$

\begin{tabular}{r|l|c|c|c||c|r|c|c|l}
\hline$v$ & $J$ & $W(\mathrm{eV})$ & \multicolumn{1}{|c|}{$\tau(\mathrm{s})$} & \multicolumn{1}{c|}{$h(\mathrm{eV})$} & $v$ & $J$ & $W(\mathrm{eV})$ & \multicolumn{1}{|c}{$\tau(\mathrm{s})$} & $h(\mathrm{eV})$ \\
\hline 0 & 8 & 0,022346 & $3,3907 \times 10^{-6}$ & 0,200724 & 0 & 11 & 0,059512 & $4,222 \times 10^{-6}$ & 0,298 \\
0 & 9 & 0,264137 & $6,034 \times 10^{-14}$ & 0,2945 & 0 & 12 & 0,301341 & $7,620 \times 10^{-13}$ & 0,40 \\
1 & 7 & 0,130137 & $2,953 \times 10^{-14}$ & 0,1312465 & 1 & 9 & 0,052283 & $9,159 \times 10^{-10}$ & 0,135 \\
3 & 2 & 0,001917 & $3,546 \times 10^{-13}$ & 0,002142 & 1 & 10 & 0,207069 & $6,465 \times 10^{-14}$ & 0,208 \\
& & 2 & 7 & 0,056088 & $2,776 \times 10^{-13}$ & 0,072 \\
\end{tabular}


sur la position énergétique des résonances par rapport à la limite de dissociation en $\mathrm{H}^{+}+\mathrm{He}$ et de moins de $25 \%$ sur les durées de vie de ceux obtenus à l'aide du programme quantique de Le Roy [15]. La partie principale de ce programme est une amélioration considérable du sousprogramme SCHRQ mis au point par Cooley-Cashion et Zare [16]. Ce programme assimile la fonction d'onde au troisième point de rebroussement, situé vers les grandes distances internucléaires, à une fonction d'Airy du deuxième ordre. La largeur de l'état est calculée dans le cadre de l'approximation J.W.K.B. Les résultats exposés dans les tableaux I et II ont été obtenus à l'aide de ce programme de calcul qui détermine simultanément les énergies des états liés, quasi-liés et les durées de vie, calculées vis-à-vis de la prédissociation. Toutefois, ce programme de calcul ne permet pas de localiser les résonances énergétiques situées au-dessus de la barrière de potentiel centrifuge.

La figure 1 montre les courbes de potentiel effectif de $\mathrm{He}^{+}$dans son état électronique fondamental pour les nombres quantiques de rotation $J=0, J=8$. Les fonctions d'onde radiales ont été également tracées pour les niveaux $v=0, J=0$ (état lié) et $v=0, J=8$ (état quasi-lié). Le tableau I reporte les positions énergétiques des états rovibroniques de $\mathrm{He} \mu^{+}$et $\mathrm{Ne} \mu^{+}$. Ces niveaux d'énergie sont repérés par rapport à la limite de dissociation de la molécule. Les valeurs positives correspondent aux états situés au-dessus de cette limite donc aux niveaux quasi-liés. Il existe 4 et 6 états quasi-liés et 22 et 33 niveaux d'énergies d'états liés. Pour ces derniers états les périodes de vibration et de rotation sont typiquement de l'ordre de $4 \times 10^{-15}$ et $1 \times 10^{-14} \mathrm{~s}$ respectivement; pour $\mathrm{He}^{+}$et $\mathrm{Ne} \mu^{+}$, les durées de vie des états quasi-liés sont comprises entre $10^{-6}$ et $10^{-14} \mathrm{~s}$ (Tableau II).

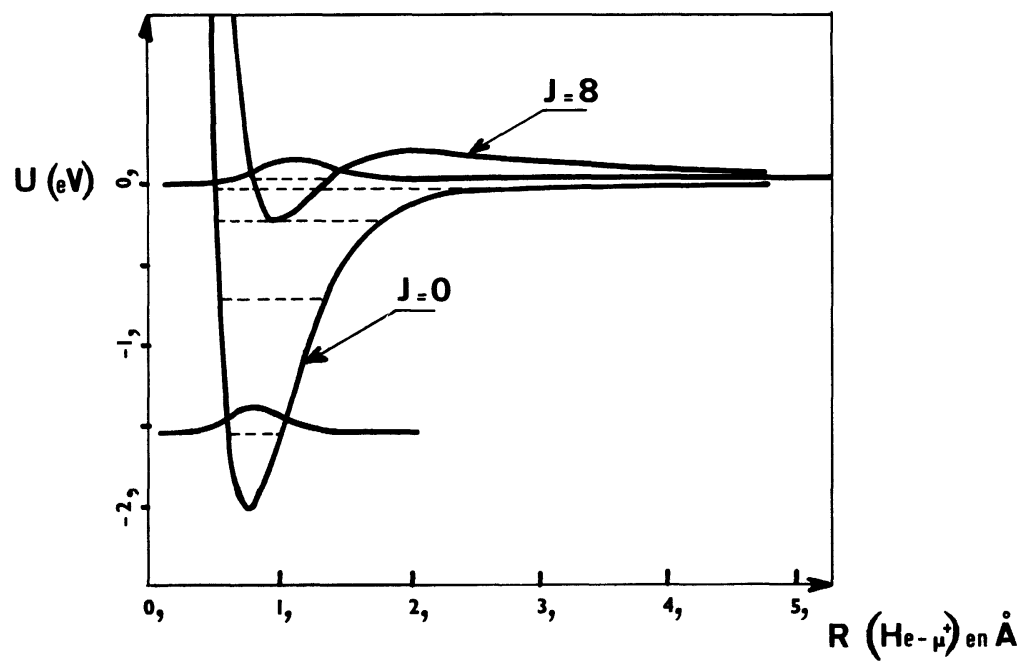

Fig. 1. - Courbes de potentiel effectif de l'ion moléculaire $\mathrm{He} \mu^{+}$pour les nombres quantiques de rotation $J=0$ et $J=8$. On a figuré les niveaux vibrationnels (traits et tirets) ainsi que les fonctions d'onde des niveaux $v=0, J=0$ et $v=0, J=8$.

[Effective potential curves of the molecular ion $\mathrm{He}^{+}$for rotational quantum numbers $J=0$ and $J=8$. The energy levels and the vibrational wave functions have been drawn for $v=0, J=0$ and $v=0, J=8$. The dashed lines indicate the vibrational energy levels.]

A l'aide d'un calcul classique, Mikula [11a] situait les niveaux $v=0$ et $v=1$ à $-1,69$ et $-1,07 \mathrm{eV}$ pour $\mathrm{He} \mu^{+}$et à $-1,73 \mathrm{et}-0,63 \mathrm{eV}$ pour $\mathrm{Ne} \mu^{+}$. Ce calcul, qui ne tient pas compte de l'énergie de rotation situe raisonnablement, avec une précision de $0,1 \mathrm{eV}$, le premier niveau de vibration, mais l'anharmonicité pour les molécules muoniques est telle que le décalage pour les seconds niveaux atteint déjà $0,3 \mathrm{eV}$. 
La précision de nos résultats dépend toutefois de la validité de la méthode de calcul, de la courbe de potentiel, de l'approximation de Born-Oppenheimer.

La précision de la méthode de calcul pour la détermination des états quasi-liés a déjà été discutée. En ce qui concerne les états liés nous avons comparé les valeurs théoriques obtenues à l'aide du précédent programme [15] aux énergies des onze transitions de $\mathrm{HeH}^{+}$observées expérimentalement par Bernath et Amaro [17] : l'accord est meilleur que $0,5 \mathrm{~cm}^{-1}$ [18]. Cet accord constitue à la fois un test de la méthode de calcul et de la précision du calcul ab initio de la courbe de potentiel (estimée à $2 \mathrm{~cm}^{-1}$ ). Par contre celle de l'état fondamental ${ }^{1} \Sigma^{+} \mathrm{de} \mathrm{NeH}^{+}$a été calculée

Tableau III. - Niveaux d'énergies en $\mathrm{eV}$, par rapport à la limite de dissociation, des états rovibrationnels de $\mathrm{Ne} \mu^{+}$pour les deux courbes de potentiel encadrant la précision des calculs théoriques. Entre parenthèses sont indiquées, en secondes, les durées de vie calculées des états quasi-liés.

[Energy levels (in eV) calculated with respect to the dissociation limit, of the rovibrational levels of $\mathrm{Ne}^{+}$, obtained using the two limiting theoretical curves. Calculated lifetimes (in seconds) are indicated in brackets.]

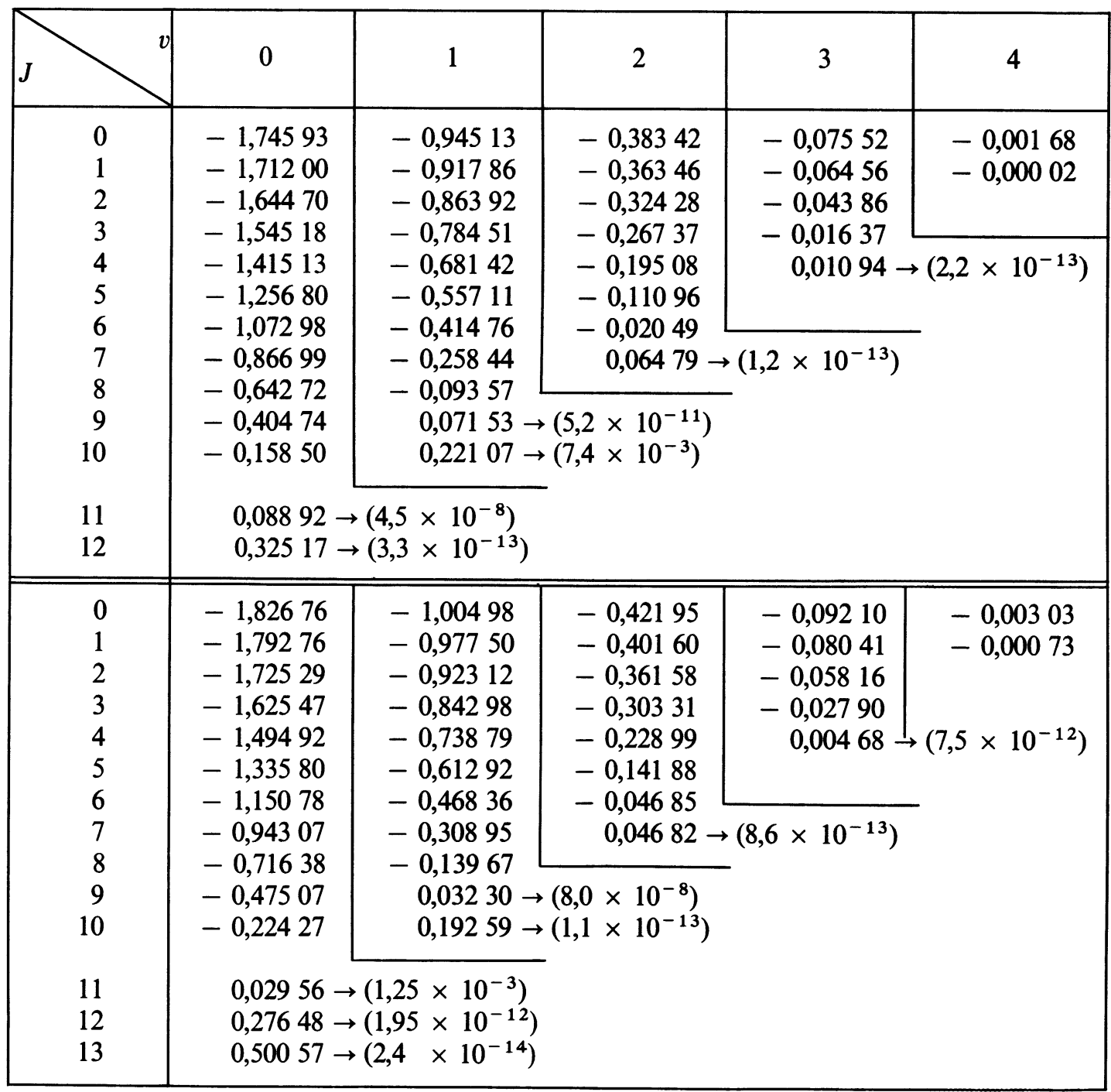


avec une incertitude de $2 \%$ à l'aide des méthodes S.C.E.P./V.A.R. et S.C.E.P./C.E.P.A. [14]. A partir de deux courbes de potentiel virtuelles, bornant la marge d'erreur de ces calculs, on a déterminé les niveaux d'énergie. Les résultats sont rassemblés dans le tableau III. La comparaison avec le tableau I montre que les fluctuations des constantes spectroscopiques sont faibles. Les positions énergétiques des états quasi-liés piégés à travers la barrière centrifuge sont déterminées avec une marge d'erreur inférieure à un facteur deux, il est remarquable que les durées de vie calculées vis-à-vis de la prédissociation varient de plusieurs ordres de grandeur : leurs éventuelles mesures expérimentales constitueraient en fait le test le plus sensible des calculs de courbe de potentiel.

La précision de la courbe de potentiel de $\mathrm{He}^{+}$est telle que l'on peut chercher à estimer l'importance des corrections dues à l'approximation de Born-Oppenheimer. Bishop, Cheung [19] et Price [20] ont calculé simultanément les corrections adiabatiques à la courbe de potentiel ${ }^{1} \Sigma$ de $\mathrm{HeH}^{+}$ainsi que les polarisabilités dipolaires dynamiques isotropes et anisotropes [21]. En choisissant une base de 255 fonctions d'onde au lieu de 83 [13], la variation de l'énergie potentielle est inférieure à $3,5 \mathrm{~cm}^{-1}$ alors que la correction adiabatique déplace les niveaux d'énergie de $8 \mathrm{~cm}^{-1}$. Cette correction adiabatique qui tient compte du couplage entre le mouvement électronique et nucléaire s'écrit $[22,23]$ pour $\mathrm{He}^{+}$:

$$
\Delta V(R)=V_{\text {B.o }}(R)-\left\langle\left(\nabla_{1}+\nabla_{2}\right)^{2}\right\rangle / 8 m-\left\langle\nabla_{R}^{2}\right\rangle / 2 m-\left\langle\nabla_{R} \cdot\left(\nabla_{1}+\nabla_{2}\right)\right\rangle / 2 M
$$

$V_{\text {B.o }}(R)$ est le potentiel calculé dans l'approximation de Born-Oppenheimer, $\nabla_{R}, \nabla_{1}$ et $\nabla_{2}$ sont relatifs respectivement aux noyaux et aux deux électrons de $\mathrm{He} \mu^{+}$repérés par rapport au centre géométrique milieu de $\mathbf{R}, m$ est la masse réduite et $M$ le facteur hétéronucléaire $\left(M=\left(m_{\mathrm{He}} \times m_{\mu}\right) /\right.$ $\left.\left(m_{\mathrm{He}}-m_{\mu}\right)\right)$.

A partir des calculs effectués dans la référence [19], pondérés par les effets de masse, on a pu déterminer les corrections adiabatiques (diagonales) pour les différentes distances internucléaires. La dépendance des termes de l'équation(1) en fonction de $R$ est illustrée sur la figure 2 . La somme de ces trois facteurs, à une constante près, a été ajoutée à la courbe de potentiel B.O [19] afin de résoudire la partie radiale de l'équation de Schrödinger. Les résultats numériques sont reportés sur le tableau IV. L'introduction de la correction adiabatique fait disparaître le niveau quasi-lié $v=1$ et $J=7$. Il est cependant difficile d'apprécier l'exactitude de cette approximation adiabatique [24] qui ne tient pas compte de la partie non adiabatique. Toutefois on peut noter que, par exemple, la correction dite diagonale rend compte de la quasi-totalité (99\%) de la partie non Born-Oppenheimer de la correction au potentiel de l'état fondamental de $\mathrm{H}_{2}$ et $\mathrm{H}_{2}^{+}$[25]. Cela paraît d'autant plus justifié dans notre cas que l'état fondamental ${ }^{1} \Sigma$ corrélé à $\mathrm{He}+\mu^{+}$ est situé à plus de $10,96 \mathrm{eV}$ du premier état électronique excité $\left({ }^{1} \Sigma^{+}\right)$corrélé à $\mathrm{He}^{+}+\mathrm{Mu}$ (rappelons que la masse du muon est deux cent sept fois supérieure à celle de l'électron).

A l'aide du calcul de perturbation de second ordre implicitement introduit dans le formalisme de Van Vleck [26], et de la relation isotopique en puissance 3/2 [27], on peut estimer que le déplacement $\Delta W$ entre deux niveaux vibrationnels sera faible et de l'ordre de $\simeq-0,2(v+1 / 2)\left(\frac{m_{\mathrm{HeH}^{+}}}{m_{\mathrm{He}^{+}}}\right)^{3 / 2}$ soit environ $4 \mathrm{~cm}^{-1}$, la correction non adiabatique pour $\mathrm{H}_{2}^{+}$étant de $-0,19(v+1 / 2) \mathrm{cm}^{-1}$. Les résultats numériques ne tiennent pas compte des corrections dues au référentiel [28] et négligent donc les facteurs liés à la translation du système de coordonnées du centre géométrique au centre de masse [29] qui dans le cas de $\mathrm{He}^{+}$se confond pratiquement avec $\mathrm{He}^{+}$. Il serait aussi possible de calculer les faibles corrections non adiabatiques dues aux couplages rotationnels, ainsi que les corrections relativistes et radiatives (Lamb Shift).

Nous avons voulu montrer qu'il existait un nombre plus important que généralement admis de niveaux rovibroniques des molécules muoniques. Les molécules muoniques, dans lesquelles un muon positif remplace un proton, sont des tests réalistes et très sensibles pour les calculs des effets de masse et ne doivent pas être confondues avec l'autre catégorie de molécules muoniques, où 


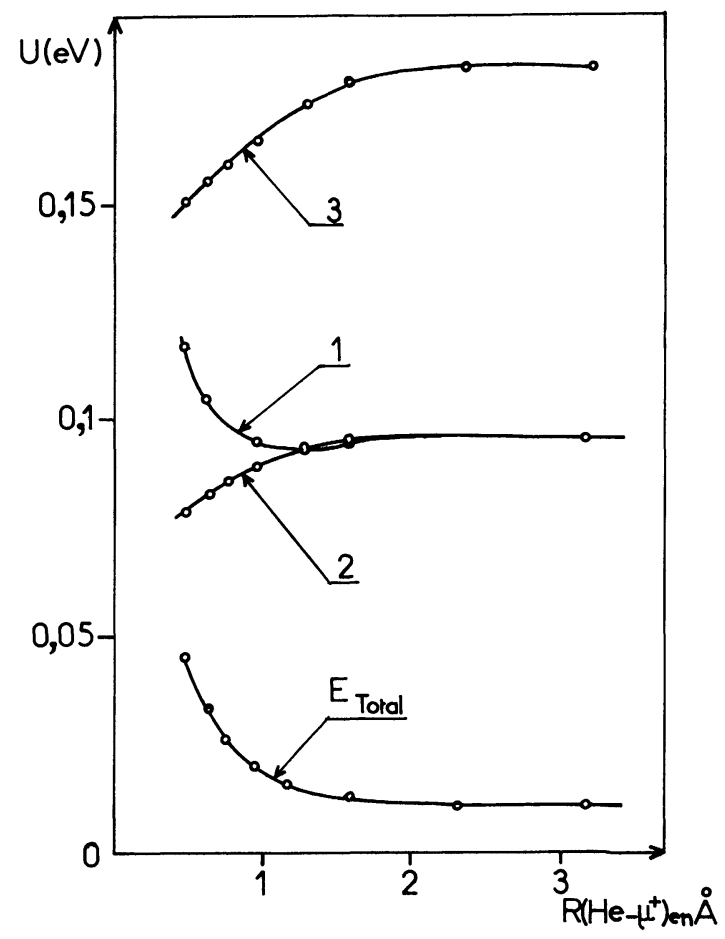

Fig. 2. - Dépendance en fonction de la distance internucléaire des termes adiabatiques de la correction à l'approximation de Born-Oppenheimer : $1=-\left\langle\left(\nabla_{1}+\nabla_{2}\right)^{2}\right\rangle / 8 m ; 2=-\left\langle\nabla_{R}^{2}\right\rangle / 2 m ; 3=\left\langle\nabla_{R}\right.$. $\left(\nabla_{1}+\nabla_{2}\right)>/ 2 M$. La courbe $E_{\text {total }}$, en eV, représente la somme de ces contributions. Pour des commodités de représentation, sur cette figure, le signe de la troisième correction a été inversé.

[Adiabatic correction terms to the Born-Oppenheimer as function of internuclear distance. The curve $E_{\text {total }}$ (in $\mathrm{eV}$ ) represents the sum of several contributions. For ease of representation, the sign of the third correction has been inverted : $\left.1=-\left\langle\left(\nabla_{1}+\nabla_{2}\right)^{2}\right\rangle / 8 m ; 2=-\left\langle\nabla_{R}^{2}\right\rangle / 2 m ; 3=\left\langle\nabla_{R} \cdot\left(\nabla_{1}+\nabla_{2}\right)\right\rangle / 2 M.\right]$

un muon négatif remplace un électron. (Kołos [30] a déjà calculé les niveaux des « isotopes muoniques électroniques » $\left(\mu^{-}\right)$de $\mathrm{H}_{2}^{+}$.)

Les états métastables ou quasi-liés peuvent être formés par recombinaison associative lors de la collision thermique du muon et de l'hélium, ou du néon. L'existence de niveaux quasi-liés de durée de vie comparable à celle du muon est intéressante : dès que le muon aura pénétré dans la barrière centrifuge, il restera piégé pendant toute son existence en tant que composant de la molécule. Les possibilités de stabilisation de ces états quasi-liés par émission infrarouge sont alors envisageables. Le niveau quasi-lié, $v=0, J=8$, dont la durée de vie calculée vis-à-vis de la prédissociation est relativement longue $\tau_{\text {pred }}=3,54 \times 10^{-7} \mathrm{~s}$, est le meilleur candidat à une éventuelle recombinaison radiative vers le niveau stable $(v=0, J=7)$ :

$$
\mu^{+}+\mathrm{He} \rightarrow \operatorname{He} \mu^{+}(v=0, J=8) \rightarrow \operatorname{He} \mu^{+}(v=0, J=7)+h v=\left(2152 \mathrm{~cm}^{-1}\right) .
$$

Le coefficient d'Einstein correspondant est

$$
A=3,137 \times 10^{-19} v^{3} \times 10^{-36}\left|\left\langle\Psi_{\mathrm{i}}|\mu| \Psi_{\mathrm{j}}\right\rangle\right|^{2}=1,4 \times 10^{5} .
$$

$A$ est en $\mathrm{s}^{-1}, v$ en $\mathrm{cm}^{-1}$, et $\mu$ est le moment dipolaire de transition exprimé en Debye, $\mu=\mu_{0}+\mu\left(R-R_{\mathrm{e}}\right)=1,66+4,29\left(R-R_{\mathrm{e}}\right)[31,32], R_{\mathrm{e}}$ est la distance d'équilibre de l'état initial $\Psi_{\text {i }}$. 
Tableau IV. - Niveaux d'énergies des états rovibrationnels de $\mathrm{He} \mu^{+}$lorsque l'on tient compte des corrections adiabatiques à l'approximation de Born-Oppenheimer. Les unités sont les mêmes que celles du tableau III. Entre parenthèses sont indiquées, en secondes, les durées de vie calculées des états quasi-liés.

[Rovibrational levels of $\mathrm{He} \mu^{+}$including adiabatic corrections to the Born-Oppenheimer approximation (units as in table III). Calculated lifetimes (in seconds) are indicated in brackets.]

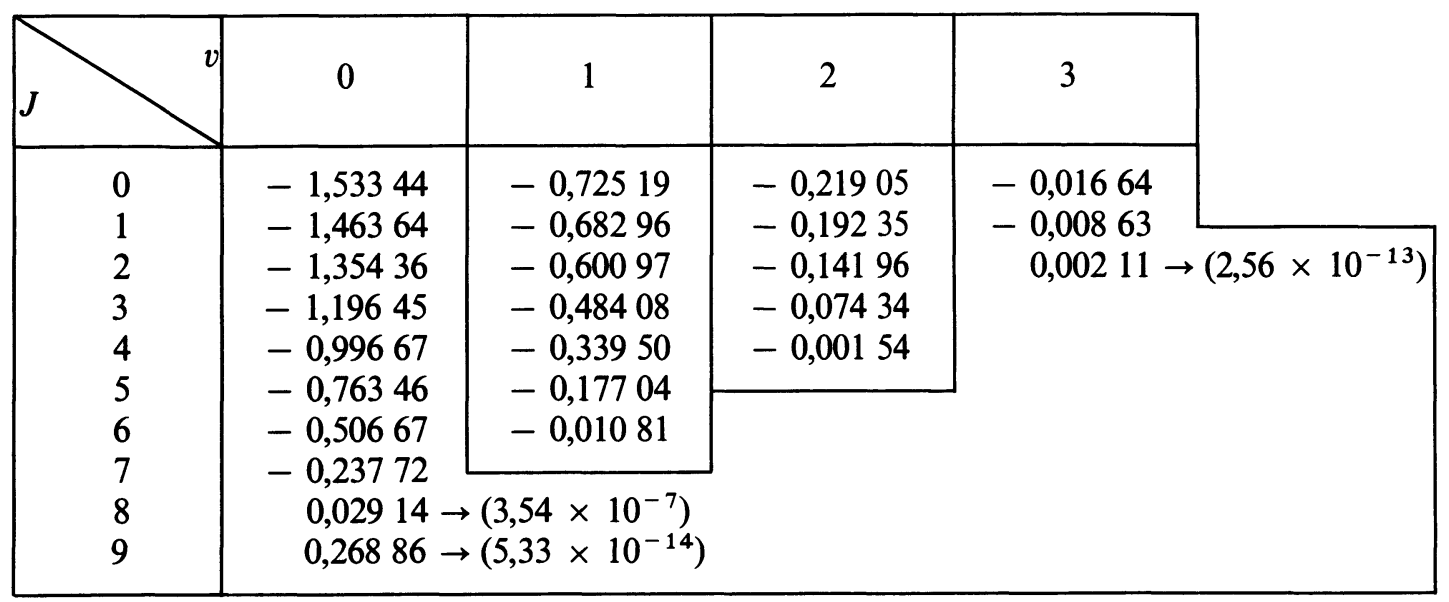

Cette durée de vie exceptionnellement courte $\left(\tau_{\text {rad }}=1 / A=7,0 \times 10^{-6} \mathrm{~s}\right)$ s'explique par la conjonction de deux facteurs : un recouvrement important des deux fonctions d'onde $(\langle\Psi \mid \Psi\rangle=0,96)$ et émission à une fréquence relativement élevée $\left(2152 \mathrm{~cm}^{-1}\right)$. En reprenant la formulation de Bain et Bardsley [33], on peut alors calculer la constante de vitesse de la réaction de formation de l'état $v=0, J=7$, accompagnée de l'émission optique précédente :

$$
\alpha=\frac{2(J+1)}{\tau_{\mathrm{pred}}+\tau_{\mathrm{rad}}} f(w)
$$

avec $J=8, w$ énergie du niveau $v=0, J=8$ par rapport à la limite de dissociation. $f(w)$ est la distribution d'énergie cinétique.

D'autre part, l'existence de nombreux états situés près de la limite de dissociation augmente de façon considérable les probabilités de formation d'ions moléculaires par stabilisation avec un $3^{\mathrm{e}}$ corps qui emporte le faible excès d'énergie [34]. De telles considérations doivent intervenir dans les études de relaxation de l'énergie cinétique des faisceaux de muons positifs. Il est possible toutefois que la formation de $\mathrm{He}^{+}$' 'effectue par l'intermédiaire de la réaction $\mathrm{Mu}+\mathrm{He}^{*} \rightarrow \mathrm{He} \mu^{+}+\mathrm{e}^{-}$, puisque dans le cas du proton, la section efficace d'un tel processus est de l'ordre de $4 \times 10^{-15} \mathrm{~cm}^{2}$ [35]. La capture directe du muonium par l'hélium pour former MuHe est violemment interdite car cette molécule est similaire à $\mathrm{HeH}$ qui est instable. Les avantages liés à l'utilisation du muon ou du muonium pour augmenter l'importance des effets isotopiques dans les calculs de dynamique réactionnelle ont été traités par Connor et al. [36].

Remerciements. - Nous sommes particulièrement reconnaissants à Messieurs Fleming et Mikula de nous avoir communiqué leurs résultats avant publication. 


\section{Bibliographie}

[1] Exotic atoms. 1st course of the International School of Physics of Exotic Atoms, Erice (1977), Fiorentini G., Torelli G., Eds.

[2] Sens, J. C., Swanson, R. A., Telegdi, V. L. et Yovanoreitch, D. D., Phys. Rev. 107 (1957) 1465.

[3] KIM, Y. N., Mesic atoms and nuclear structure (North Holland) 1971.

[4] Hughes, V. W., McCalm, P. W., Ziock, K. et Prepost, R., Phys. Rev. Lett. 5 (1960) 63.

[5] Fleming, D. G., Garner, D. M., Vaz, L. C., Walter, D. C., Brewer, J. H. et Crowe, K. W., dans " Positronium and muonium chemistry », Ache H. J., Ed., Adv. in Chem. Ser. no 175, page 279.

[6] Ansel'm, A. A. et Shekhter, V. M., Sov. Phys. JETP 16 (1963) 678.

[7] a) Brouard, J., Comtet, G., Frohlich, H. et Fournier, P. G., 10e ICPEAC Paris (1977) 598.

b) Fournier, P. G., Lock, R., MaAs, J., Van Asselt, N., Los, J., Chem. Phys. Lett. 40 (1976) 170.

[8] Mikula, R. J., Garner, D. M., Marshall, G. M., Brewer, J. H., Hyperfine Interactions 6 (1979) 379 et

FLEMING, D. G., 50 years dynamics of chemical reaction, Berlin (1981) et communication privée.

[9] Crane, T. W., Casperson, D. E., Chang, H., Hughes, V. W., Kaspar, H. F., Lovett, B., Schiz, A., Souder, P., Stambough, R. D., Zu Putlitz, G., Phys. Rev. Lett. 33 (1974) 572.

[10] Stambough, R. D., Casperson, D. E., Crane, T. W., Hughes, V. W., Kaspar, H. F., Souder, P., Thompson, P. A., Orth, H., Zu Putlitz, G., Dennison, A. B., Phys. Rev. Lett. 33 (1974) 568.

[11] a) MikUla, R. J., Ph. D. Thesis, University of British Columbia (Août 1981).

b) Fleming, D. G., Mikula, R. J., GaRneR, D. M., Hyperfine Interactions 9 (1981) 207.

[12] ChILD, M. S., Molecular Spectroscopy II. A specialist periodical report of the Chemical Society (London), edited by Barrow, R. F., Long, D. A. and Millen, D. J. (Chemical Society, London) 1974, p. 466.

[13] a) Kozos, W., Peek, J. M., Chem. Phys. 12 (1976) 381.

b) Kozos, W., Int. J. Quant. Chem. 10 (1976) 217.

[14] Rosmus, P., ReInsh, E. A., Z. Naturforsch. 35 (1980) 1066.

[15] Le Roy, R. J., LiU, W. K., J. Chem. Phys. 69 (1978) 3622.

[16] a) CoOley, J. W., Math. Comput. 15 (1961) 363.

b) Cashion, J. K., J. Chem. Phys. 38 (1963) 1872.

c) ZARE, R. N., University of California Radiation Laboratory Report UCRL. 10925 (1963).

[17] Bernath, P., Amono, T., Phys. Rev. Lett. 48 (1982) 20.

[18] Fournier, P. G., Lassier-Govers, B., Brouard, J., Comtet, G., ge Colloque sur la Physique des collisions atomiques et moléculaires, Louvain (1980).

[19] Bishop, D. M., Cheung, L. M., J. Mol. Spectrosc. 75 (1979) 462.

[20] PRICE, R. I., Chem. Phys. 31 (1978) 309.

[21] Bishop, D. M., Cheung, L. M., J. Chem. Phys. 72 (1980) 5125.

[22] Kolos, W., Wolniewicz, L., Rev. Mod. Phys. 35 (1963) 463.

[23] Kołos, W., Wolniewicz, L., Chem. Phys. 41 (1964) 3663.

[24] Kozos, W., Adv. Quant. Chem. 5 (1970) 99.

[25] Kokos, W., Wolniewicz, L., J. Mol. Spectrosc. 54 (1975) 303.

[26] Van VleCK, J. Chem. Phys. 4 (1936) 327.

[27] Bishop, D. M., Mol. Phys. 28 (1974) 1397.

[28] Thorson, W. R., Delos, J. B., Phys. Rev. A 18 (1978) 117.

[29] Les "subtilités" liées aux calculs du facteur de translation sont explicitées dans RiERA, A. et Salin, A., J. Phys. B 9 (1976) 2877 et

Salin, A., Comments At. Mol. Phys. 9 (1980) 165.

[30] Kołos, W., Acta Phys. Acad. Sci. Hung. 27 (1969) 241.

[31] Peyerimhoff, S., J. Chem. Phys. 44 (1965) 998.

[32] Michels, H. H., J. Chem. Phys. 44 (1966) 3834.

[33] Bain, R. A., Bardsley, J. N., J. Phys. B : Atom. Mol. Phys. 5 (1972) 277.

[34] Aux énergies thermiques la probabilité de dissociation du complexe $\mathrm{He} \mu^{+}$par collision sur un troisième corps est très inférieure à la probabilité de stabilisation à trois corps $(\Delta t \ll 2 \mu \mathrm{s})$.

[35] Fort, J. C., Laucagne, J. J., Pesnelle, A., Watel, G., Phys. Rev: 14 (1976) 659.

[36] Connor, J. N. L., Lagana, A., Turfa, A. F., Whitehead, J. C., J. Chem. Phys. 75 (1981) 3301. 